\title{
Analyzing the Boer-Mulders function within different quark models
}

\author{
A. Courtoy ${ }^{1 *}$, S. Scopetta ${ }^{2 \dagger}$, V. Vento ${ }^{1 \ddagger}$ \\ (1) Departament de Fisica Teòrica, \\ Universitat de València \\ and Institut de Fisica Corpuscular, \\ Consejo Superior de Investigaciones Científicas \\ 46100 Burjassot (València), Spain \\ (2) Dipartimento di Fisica, \\ Università degli Studi di Perugia, and \\ INFN, sezione di Perugia, \\ via A. Pascoli 06100 Perugia, Italy
}

\begin{abstract}
A general formalism for the evaluation of time reversal odd parton distributions is applied here to calculate the Boer-Mulders function. The same formalism when applied to evaluate the Sivers function led to results which fulfill the Burkardt sum rule quite well. The calculation here has been performed for two different models of proton structure: a constituent quark model and the MIT bag model. In the latter case, important differences are found with respect to a previous evaluation in the same framework, a feature already encountered in the calculation of the Sivers function. The results obtained are consistent with the present wisdom, i.e., the contributions for the $u$ and $d$ flavors turn out to have the same sign, following the pattern suggested analyzing the model independent features of the impact parameter dependent generalized parton distributions. It is therefore confirmed that the present approach is suitable for the analysis of time reversal odd distribution functions. A critical comparison between the outcomes of the two models, as well as between the results of the calculations for the Sivers and Boer-Mulders functions, is also carried out.
\end{abstract}

\footnotetext{
* E-mail address: aurore.courtoy@uv.es

$\dagger$ E-mail address: sergio.scopetta@pg.infn.it

$\ddagger$ E-mail address: vicente.vento@uv.es
} 


\section{INTRODUCTION}

The study of the transverse polarization of quarks in the nucleon, one of its less known features (for a review, see, e.g., Ref. [1]), is progressing fast, since the expected new data from ongoing experiments are motivating a strong theoretical activity (for recent developments, see Ref. [2]). The present work aims to contribute to the understanding of the transverse polarization of quarks by evaluating, using different models of the proton structure, the Boer-Mulders function [3]. We will use a theoretical scenario [4, 5] recently developed for the calculation of the Sivers function [6], which has reproduced its main features, such as the sign, the Burkardt sum rule [7], and the general trend of the parameterizations extracted from the available data [8].

The Boer-Mulders function $h_{1}^{\perp}$ describes the number density of transversely polarized quarks in an unpolarized target. As the Sivers function, $f_{1 T}^{\perp}$, describing the number density of unpolarized quarks in a transversely polarized target, the Boer-Mulders function is a Transverse Momentum Dependent (TMD) PD and it is a time-reversal odd object (T-odd) [1]. For this reason, for several years, it was believed to vanish due to time reversal invariance. However, this argument was invalidated, initially in the case of the Sivers function, by a

calculation in a spectator model [9], following the observation of the existence of leadingtwist Final State Interactions (FSI) [10]. The current wisdom is that non-vanishing T-odd functions are generated by the gauge link in the definition of TMD parton distributions [11, 12, 13], whose contribution does not vanish in the light-cone gauge, as it happens for the standard PD functions. Those T-odd functions can be intuitively related to impact parameter dependent (IPD) parton distributions [14, 15]. However, because of the gauge link, it is formally questionable. For example, in the case of the Sivers function, it has been shown that a model independent relation between this quantity and the corresponding GPD is still to be found [16].

The Boer-Mulders function is being measured through Semi-inclusive deep inelastic scattering (SIDIS) [17, 18, 19] (see Ref. [20] for an analysis of these data) and through the Drell-Yan (DY) process in hadronic colliders [21, 22, 23]. In particular, in Ref. [24], the Boer-Mulders function has been recently extracted from the data of the unpolarized $p+D$ Drell-Yan processes measured by the E866/NuSea Collaboration at FNAL [22]. However, the extraction of $h_{1}^{\perp}$ is very difficult, as it always involves another chirally-odd distribution 
function. As a matter of fact, in SIDIS, the Collins fragmentation function is required [25], and in DY the observable quantity is a convolution of two $h_{1}^{\perp}$ belonging to the two colliding nucleons [26]. Presently, the lack of accurate data affects the quality of the extraction.

The present experimental scenario motivates therefore the formulation of theoretical estimates. In principle one should perform a calculation in QCD; however, this is presently not possible. Lacking this possibility, it becomes relevant to perform model calculations of the Boer-Mulders function. A few estimates exist. In a quark-diquark model with axial vector diquarks, it was originally found that $h_{1}^{\perp}$ has a different sign for the $u$ and $d$ flavors [27]. These findings have been corrected by the Authors of Ref. [28], who, following a procedure established in previous papers [29], demonstrated that the sign of $h_{1}^{\perp}$ for the $u$ and $d$ flavors turns out to be the same, assuming the spectator diquark to be either scalar or axial vector. This feature is in qualitative agreement with the pattern predicted by quark helicity-flip IPD GPDs in models [30, 31] and in lattice simulations [32]. It has also been found in a few other model calculations: in the MIT bag model, in its simplest version [33]; in a large $N_{c}$ analysis of TMDs [34]; in a phenomenological parameterization based on the quark-diquark picture [35].

In here, the recently proposed formalism of Refs. [4] and [5], used so far for the evaluation of the Sivers function in a Constituent Quark Model (CQM) and in the MIT bag model, respectively, will be extended to calculate $h_{1}^{\perp}$ for the valence quarks. In the case of the Sivers function, within both models, this approach has proven to be able to reproduce the main features of $f_{1 T}^{\perp}$. Similar expectations motivate the present analysis of $h_{1}^{\perp}$. The MIT bag model calculation presented here has an additional purpose, namely completeness. As a matter of fact, in Ref. [33], the general framework for the calculation of T-odd TMDs has been nicely set up, but an important contribution has been nevertheless disregarded. In Ref. [5], we have reincorporated this contribution into the calculation of the Sivers function. We found that the results of Ref. [33] were incomplete and, as a consequence, they did not fulfill the Burkardt Sum Rule. Once this missing contribution is properly included in the calculation, the Burkardt Sum Rule turns out to be fulfilled to a large extent [5]. The same problem affects the evaluation of $h_{1}^{\perp}$ in Ref. [33], which is therefore retaken here, along the lines of Ref. [5].

The paper is structured as follows. In the second section, the main quantities of interest are introduced and the formalism for the calculation of $h_{1}^{\perp}$ in a CQM and in the MIT bag 
model is developed (some technical details are given in the Appendix). The numerical results of the calculations are presented and discussed in the following section. In the last section, we draw conclusions from our study. All throughout the paper, the formalism and the results are critically compared with those previously obtained for the Sivers function $f_{1 T}^{\perp}$.

\section{FORMALISM}

The Boer-Mulders function, $h_{1}^{\perp \mathcal{Q}}\left(x, k_{T}\right)$ [3], the quantity of interest here, is formally defined, according to the Trento convention [36], for the quark of flavor $\mathcal{Q}$, through the following expression ${ }^{1}$ :

$$
\begin{aligned}
h_{1}^{\perp \mathcal{Q}}\left(x, k_{T}\right) & =-\frac{M}{2 k_{x}} \int \frac{d \xi^{-} d^{2} \vec{\xi}_{T}}{(2 \pi)^{3}} e^{-i\left(x p^{+} \xi^{-}-\vec{k}_{T} \cdot \vec{\xi}_{T}\right)} \\
& \times \frac{1}{2} \sum_{S_{z}=-1,1}\left\langle P S_{z}\left|\bar{\psi}_{\mathcal{Q}}\left(\xi^{-}, \vec{\xi}_{T}\right) \mathcal{L}_{\vec{\xi}_{T}}^{\dagger}\left(\infty, \xi^{-}\right) \gamma^{+} \gamma^{2} \gamma_{5} \mathcal{L}_{0}(\infty, 0) \psi_{\mathcal{Q}}(0,0)\right| P S_{z}\right\rangle+\text { h.c. },
\end{aligned}
$$

where $\vec{S}$ is the spin of the target hadron, the normalization of the covariant spin vector is $S^{2}=-1, M$ is the target mass, $\psi_{\mathcal{Q}}(\xi)$ is the quark field and the gauge link is

$$
\mathcal{L}_{\vec{\xi}_{T}}\left(\infty, \xi^{-}\right)=\mathcal{P} \exp \left(-i g \int_{\xi^{-}}^{\infty} A^{+}\left(\eta^{-}, \vec{\xi}_{T}\right) d \eta^{-}\right)
$$

where $g$ is the strong coupling constant. One should notice that this definition for the gauge link holds in covariant (non singular) gauges, and in SIDIS processes, since the definition of T-odd TMDs is process dependent. As observed in Ref. [9] for the first time, and later in [37, 38] using factorization theorems, the gauge link contains a scaling contribution which makes the T-odd TMDs non vanishing in the Bjorken limit.

For completeness, we recall now the definition of the Sivers function, $f_{1 T}^{\perp \mathcal{Q}}\left(x, k_{T}\right)$. Taking

\footnotetext{
${ }^{1}$ An equivalent expression is obtained by performing the following changes in Eq. (1): $k_{x} \rightarrow-k_{y}$ and $\gamma^{2} \rightarrow \gamma^{1}$. Here and in the following, $a^{ \pm}=\left(a_{0} \pm a_{3}\right) / \sqrt{2}$ and $k_{T}=\left|\vec{k}_{T}\right|$.
} 
the proton polarized along the $y$ axis, one has

$$
\begin{aligned}
f_{1 T}^{\perp \mathcal{Q}}\left(x, k_{T}\right) & =-\frac{M}{2 k_{x}} \int \frac{d \xi^{-} d^{2} \vec{\xi}_{T}}{(2 \pi)^{3}} e^{-i\left(x p^{+} \xi^{-}-\vec{k}_{T} \cdot \vec{\xi}_{T}\right)} \\
& \times \frac{1}{2} \sum_{S_{y}=-1,1} S_{y}\left\langle P S_{y}\left|\bar{\psi}_{\mathcal{Q}}\left(\xi^{-}, \vec{\xi}_{T}\right) \mathcal{L}_{\vec{\xi}_{T}}^{\dagger}\left(\infty, \xi^{-}\right) \gamma^{+} \mathcal{L}_{0}(\infty, 0) \psi_{\mathcal{Q}}(0,0)\right| P S_{y}\right\rangle+\text { h.c. }
\end{aligned}
$$

The difference between the two quantities is clearly seen and physically transparent in Eqs. (11) and (3). The function $h_{1}^{\perp \mathcal{Q}}\left(x, k_{T}\right)$ counts the transversely polarized quarks (as given by the Dirac Operator $\gamma^{+} \gamma^{2} \gamma_{5}$ in Eq. (1)) in an unpolarized proton (as given by the average over the proton helicity $S_{z}$ in Eq. (1) ), while $f_{1 T}^{\perp \mathcal{Q}}\left(x, k_{T}\right)$ counts the unpolarized quarks (as given by the Dirac Operator $\gamma^{+}$in Eq. (3) ) in a transversly polarized proton (as given by the explicit transverse component $S_{y}$ in Eq. (3)). If there were no gauge links, the two T-odd functions $f_{1 T}^{\perp \mathcal{Q}}\left(x, k_{T}\right)$ and $h_{1}^{\perp \mathcal{Q}}\left(x, k_{T}\right)$ would be identically zero. Expanding the gauge link to the first non-trivial order, i.e. the next to leading one, the Boer-Mulders function $h_{1}^{\perp \mathcal{Q}}\left(x, k_{T}\right)$, Eq. (11), becomes

$$
\begin{aligned}
h_{1}^{\perp \mathcal{Q}}\left(x, k_{T}\right) & =-\frac{M}{2 k_{x}} \int \frac{d \xi^{-} d^{2} \vec{\xi}_{T}}{(2 \pi)^{3}} e^{-i\left(x p^{+} \xi^{-}-\vec{k}_{T} \cdot \vec{\xi}_{T}\right)} \\
& \times \frac{1}{2} \sum_{S_{z}=-1,1}\left\langle P S_{z}\right| \bar{\psi}_{\mathcal{Q} i}\left(\xi^{-}, \vec{\xi}_{T}\right) \\
& \times(i g) \int_{\xi^{-}}^{\infty} A_{a}^{+}\left(\eta^{-}, \vec{\xi}_{T}\right) d \eta^{-} T_{i j}^{a} \gamma^{+} \gamma^{2} \gamma_{5} \psi_{\mathcal{Q} j}(0,0)\left|P S_{z}\right\rangle+\text { h.c. }
\end{aligned}
$$

The formalism will be presented now for the evaluation of $h_{1}^{\perp \mathcal{Q}}\left(x, k_{T}\right)$ in the MIT bag model and in the CQM. Let us start from the former. At the beginning, our procedure will follow step by step the one nicely set up in Ref. [33] for the evaluation of T-odd TMDs in the MIT bag model. The properly normalized fields for the quark in the bag (see also [39]) are given in terms of the quark wave function in momentum space, which read

$$
\varphi_{m}(\vec{k})=i \sqrt{4 \pi} N R_{0}^{3}\left(\begin{array}{c}
t_{0}(|\vec{k}|) \chi_{m} \\
\vec{\sigma} \cdot \hat{k} t_{1}(|\vec{k}|) \chi_{m}
\end{array}\right)
$$

with the normalization factor $N$

$$
N=\left(\frac{\omega^{3}}{2 R_{0}^{3}(\omega-1) \sin ^{2} \omega}\right)^{1 / 2} ;
$$


where $\omega=2.04$ for the lowest mode and $R_{0}=4 \omega / M$ is the bag radius. The functions $t_{i}(k)$ are defined as

$$
t_{i}(k)=\int_{0}^{1} u^{2} d u j_{i}\left(u k R_{0}\right) j_{i}(u \omega)
$$

Following Ref. [33], we fix the other ingredients of Eq. (44), having in mind Fig. 1 (and its h.c.). In particular, the gluon propagator is treated in a perturbative way and it is assumed that it is not modified in the bag medium. This leads, in the Landau gauge, to

$$
\begin{aligned}
h_{1}^{\perp \mathcal{Q}}\left(x, k_{T}\right) & =\frac{g^{2} M E_{P}}{k_{x}} \int \frac{d^{3} k_{3}}{(2 \pi)^{3}} \frac{d^{3} k_{1}}{(2 \pi)^{3}} \frac{d^{4} q}{(2 \pi)^{4}} \delta\left(k_{1}^{+}-x P^{+}+q^{+}\right) \delta^{(2)}\left(\vec{k}_{1 T}-\vec{k}_{T}+\vec{q}_{T}\right) \\
& \times 2 \pi \delta\left(q_{0}\right) \frac{1}{q^{+}+i \epsilon} \frac{1}{q^{2}+i \epsilon} \\
& \times \frac{1}{2} \sum_{S_{z}=-1,1} \sum_{\beta} \sum_{m_{1}, m_{2}, m_{3}, m_{4}} T_{i j}^{a} T_{k l}^{a}\left\langle P S_{z}\left|b_{\mathcal{Q} m_{1}}^{i \dagger} b_{\mathcal{Q} m_{2}}^{j} b_{\beta m_{3}}^{k \dagger} b_{\beta m_{4}}^{l}\right| P S_{z}\right\rangle \\
& \times \varphi_{m_{1}}^{\dagger}\left(\vec{k}_{1}\right) \gamma^{0} \gamma^{+} \gamma^{2} \gamma_{5} \varphi_{m_{2}}(\vec{k}) \varphi_{m_{3}}^{\dagger}\left(\vec{k}_{3}\right) \gamma^{0} \gamma^{+} \varphi_{m_{4}}\left(\vec{k}_{3}-\vec{q}\right)+\text { h.c. } .
\end{aligned}
$$

The last expression corresponds to the definition of the Boer-Mulders function in the MIT bag model, given in Ref. [33].

Substituting in Eq. (7) the identity

$$
\frac{1}{q^{+}-i \epsilon}-\frac{1}{q^{+}+i \epsilon}=i 2 \pi \delta\left(q^{+}\right)
$$

and performing the trivial integrations, one gets

$$
\begin{aligned}
h_{1}^{\perp \mathcal{Q}}\left(x, k_{T}\right) & =-2 i g^{2} \frac{M E_{P}}{k_{x}} \int \frac{d^{2} \vec{q}_{T}}{(2 \pi)^{5}} \frac{1}{q^{2}} \\
& \times \frac{1}{2} \sum_{S_{z}=-1,1} \sum_{\beta} \sum_{m_{1}, m_{2}, m_{3}, m_{4}} T_{i j}^{a} T_{k l}^{a}\left\langle P S_{z}\left|b_{\mathcal{Q} m_{1}}^{i \dagger} b_{\mathcal{Q} m_{2}}^{j} b_{\beta m_{3}}^{k \dagger} b_{\beta m_{4}}^{l}\right| P S_{z}\right\rangle \\
& \times \varphi_{m_{1}}^{\dagger}\left(\vec{k}-\vec{q}_{T}\right) \gamma^{0} \gamma^{+} \gamma^{2} \gamma_{5} \varphi_{m_{2}}(\vec{k}) \int \frac{d^{3} k_{3}}{(2 \pi)^{3}} \varphi_{m_{3}}^{\dagger}\left(\vec{k}_{3}\right) \gamma^{0} \gamma^{+} \varphi_{m_{4}}\left(\vec{k}_{3}-\vec{q}_{T}\right)
\end{aligned}
$$

The last line here depends on the four spin indices, whose combinations are dictated by the spin-flavor-color matrix elements given in the second line. Let us see now where the differences between the present calculation and that of Ref. [33] arise. Following the notation of Ref. [5], we write

$$
\varphi_{m_{1}}^{\dagger}\left(\vec{k}-\vec{q}_{T}\right) \gamma^{0} \gamma^{+} \gamma^{2} \gamma_{5} \varphi_{m_{2}}(\vec{k})=I_{m_{1}}\left(\vec{k}, \vec{q}_{T}\right) \delta_{m_{1} m_{2}}+J_{m_{1}}\left(\vec{k}, \vec{q}_{T}\right) \delta_{m_{1},-m_{2}}
$$




$$
\int \frac{d^{3} k_{3}}{(2 \pi)^{3}} \varphi_{m_{3}}^{\dagger}\left(\vec{k}_{3}\right) \gamma^{0} \gamma^{+} \varphi_{m_{4}}\left(\vec{k}_{3}-\vec{q}_{T}\right)=F_{m_{3}}\left(\vec{q}_{T}\right) \delta_{m_{3} m_{4}}+H_{m_{3}}\left(\vec{q}_{T}\right) \delta_{m_{3},-m_{4}}
$$

In these expressions, all the possible helicity combinations in the initial and final states are emphasized. To our understanding, there are no physical reasons to rule out none of the above possible contributions. It is easily realized that both the terms with no-helicity flip of the quark 3, i.e. $F_{m_{3}}$, as well as the one allowing the helicity-flip of this quark, i.e. $H_{m_{3}}$, are non-vanishing under the integration over $d^{3} k_{3}$. This result is in contrast with the result presented in Ref. [33], where only a term similar to $F_{m_{3}}$ is considered. As we explained in Ref. [5], this result only applies if the integral is performed taking $\vec{q}_{T}$ along the $z$ direction. However, this is incorrect because, in any DIS process, the operator structure is determined by the direction of the virtual photon. Here, the operator $\gamma^{+}$results from having taken the photon along the $z$ axis. Therefore we no longer have the freedom to choose $z$ as the direction of the exchanged gluon, which must then lie in the $(x, y)$ plane. Besides, one can check that the integral Eq. (10) does depend on the direction of $\vec{q}_{T}$. Moreover, if the findings of Ref. [33] were correct, it would mean that no helicity flip for the interacting quark could occur, a restriction which does not have any physical motivation. Thus the present calculation differs from the previous one in that we take into consideration both terms of Eq. (10). By the same argument, the expression $\varphi_{m_{1}}^{\dagger}\left(\vec{k}-\vec{q}_{T}\right) \gamma^{0} \gamma^{+} \gamma^{2} \gamma_{5} \varphi_{m_{2}}(\vec{k})$ in Eq. (9) also contains both helicity-flip and non-flip terms.

Using Eqs. (9) and (10) in Eq. (8), the Boer-Mulders function reads

$$
\begin{aligned}
h_{1}^{\perp \mathcal{Q}}\left(x, k_{T}\right) & =-2 i g^{2} \frac{M E_{P}}{k_{x}} \int \frac{d^{2} q_{T}}{(2 \pi)^{5}} \frac{1}{q^{2}} \\
& \times \frac{1}{2} \sum_{S_{z}=-1,1} \sum_{\beta} \sum_{m_{1}, m_{2}, m_{3}, m_{4}} T_{i j}^{a} T_{k l}^{a}\left\langle P S_{z}\left|b_{\mathcal{Q} m_{1}}^{i \dagger} b_{\mathcal{Q} m_{2}}^{j} b_{\beta m_{3}}^{k \dagger} b_{\beta m_{4}}^{l}\right| P S_{z}\right\rangle \\
& \times\left\{I_{m_{1}}\left(\vec{k}, \vec{q}_{T}\right) \delta_{m_{1} m_{2}}+J_{m_{1}}\left(\vec{k}, \vec{q}_{T}\right) \delta_{m_{1},-m_{2}}\right\}\left\{F_{m_{3}}\left(\vec{q}_{T}\right) \delta_{m_{3} m_{4}}+H_{m_{3}}\left(\vec{q}_{T}\right) \delta_{m_{3},-m_{4}}\right\} .
\end{aligned}
$$

After the evaluation of the spin-flavor-color matrix elements,

$$
\sum_{\beta} T_{i j}^{a} T_{k l}^{a}\left\langle P S_{z}\left|b_{\mathcal{Q} m_{1}}^{i \dagger} b_{\mathcal{Q} m_{2}}^{j} b_{\beta m_{3}}^{k \dagger} b_{\beta m_{4}}^{l}\right| P S_{z}\right\rangle=C_{\mathcal{Q} S_{z}}^{m_{1} m_{2}, m_{3} m_{4}}
$$

performed assuming $\mathrm{SU}(6)$ symmetry, for $\mathcal{Q}=u, d$ and $S_{z}=1(-1)$ (these coefficients are listed in the Appendix), and after a straightforward calculation, one is left with the final 
expression

$$
\begin{aligned}
h_{1}^{\perp u(d)}\left(x, k_{T}\right) & =-g^{2} \frac{M E_{P}}{k^{x}} \int \frac{d^{2} q_{T}}{(2 \pi)^{2}} \frac{C^{2}}{q^{2}} \\
& \times\left\{6(3) I_{1} F_{1}+1(2) I_{2} F_{2}+1(2) J_{1} H_{1}+1(2) J_{2} H_{2}\right\},
\end{aligned}
$$

with the eight functions $I_{1,2}, F_{1,2}, H_{1,2}, J_{1,2}$ and the $c$-number $C$ given in the Appendix. The two first terms on the r.h.s. of (13) are the contributions involving quarks which do not flip helicity. On the other hand, the last two terms on the r.h.s. of the same expression represent the contribution due to the double helicity flip of the quarks. This contribution will turn out to be non negligible. We reiterate that the result Eq. (13) is different from that of the previous calculation in the bag model [33], which includes only a $F_{m_{3}}$-like term.

Through the coefficients Eq. (12) it is possible to reconstruct what happens at the level of the quark helicity in a perfectly transparent way. The rôle of each contribution can be followed and evaluated. The dominant contributions are the non-flipping $I_{1} F_{1}$ and the double-flipping $J_{1} H_{1}$ ones. Due to the spin-flavor-color coefficients, i.e., due to the $\mathrm{SU}(6)$ symmetry assumption, the non-flipping term is bigger than the double-flipping contribution. The improvement of the Boer-Mulders in the MIT bag model with respect to the previous calculation [33] quantitatively consists in the addition of the double-flipping contribution. In effect, the other two terms, $I_{2} F_{2}$ and $J_{2} H_{2}$, are governed by the product of the two lower components of the bag wave function (cf. Eqs. (A8) and (A14) in the Appendix), which encodes the most relativistic contribution arising in the MIT bag model. They turn out to be a few orders of magnitude smaller than the dominant ones, arising from the interference between the upper and lower parts of the bag wave function. We will see now that this happens also if a proper non relativistic reduction (NR) of the gauge link, suitable for CQM calculations, is performed.

The calculation scheme for T-odd functions in a CQM has been completely set up in Ref. [4], where it has been applied to the Sivers function. Since the formalism required here for the Boer-Mulders function is the same used in our previous paper to evaluate $f_{1}^{\perp \mathcal{Q}}\left(x, k_{T}\right)$, we refer to that paper to obtain a workable formula for $h_{1}^{\perp \mathcal{Q}}\left(x, k_{T}\right)$. As it has been discussed after Eqs. (1) and (3), with respect to the $f_{1}^{\perp \mathcal{Q}}\left(x, k_{T}\right)$ calculation, one has to change the polarization of the proton and the Dirac structure of the quark operator. Starting from the definition, Eq. (1), it is found therefore that, in a CQM, the equivalent of the expression 
Eq. (8), previously obtained in the MIT bag model, is:

$$
h_{1}^{\perp \mathcal{Q}}\left(x, k_{T}\right)=-2 i g^{2} \frac{M^{2}}{k_{x}} \int d \vec{k}_{1} d \vec{k}_{3} \frac{d^{2} \vec{q}_{T}}{(2 \pi)^{2}} \delta\left(k_{1}^{+}-x P^{+}\right) \delta\left(\vec{k}_{1 T}+\vec{q}_{T}-\vec{k}_{T}\right) \mathcal{M}^{\mathcal{Q}}
$$

where

$$
\begin{aligned}
\mathcal{M}^{\mathcal{Q}} & =\sum_{\mathcal{F}_{3}, m_{1}, c_{3}, m_{2}, c_{4}, m_{3}, i, m_{4}, j} \delta_{\left(S_{z}, r, m_{1}, m_{2}, l_{n}, m_{3}, m_{4}, i, j, c_{3}, c_{4}\right)} \frac{1}{2} \sum_{S_{z}=-1,1} \\
& \times \Psi_{r S_{z}}^{\dagger}\left(\vec{k}_{1}\left\{m_{1}, i, \mathcal{Q}\right\} ; \vec{k}_{3}\left\{m_{3}, c_{3}, \mathcal{F}_{3}\right\} ;-\vec{k}_{3}-\vec{k}_{1}, l_{n}\right) \\
& \times T_{i j}^{a} T_{c_{3} c_{4}}^{a} V^{h}\left(\vec{k}_{1}, \vec{k}_{3}, \vec{q}\right) \\
& \times \Psi_{r S_{z}}\left(\vec{k}_{1}+\vec{q},\left\{m_{2}, j, \mathcal{Q}\right\} ; \vec{k}_{3}-\vec{q},\left\{m_{4}, c_{4}, \mathcal{F}_{3}\right\} ;-\vec{k}_{3}-\vec{k}_{1}, l_{n}\right),
\end{aligned}
$$

where, $l_{i}=\left\{m_{i}, c_{i}, \mathcal{Q}\right\}$ represents the set of helicity, color and flavor quantum numbers, respectively, and the vector $\Psi_{r S_{z}}$ is an intrinsic proton state. Given the diagram shown in Fig. 1, the interaction $V^{h}\left(\vec{k}_{1}, \vec{k}_{3}, \vec{q}\right)$ reads

$$
V^{h}\left(\vec{k}_{1}, \vec{k}_{3}, \vec{q}\right)=\frac{1}{q^{2}} \bar{u}_{m_{1}}\left(\vec{k}_{1}\right) \gamma^{+} \gamma^{2} \gamma_{5} u_{m_{2}}\left(\vec{k}_{1}+\vec{q}\right) \bar{u}_{m_{3}}\left(\vec{k}_{3}\right) \gamma^{+} u_{m_{4}}\left(\vec{k}_{3}-\vec{q}\right)
$$

$\Psi_{r S_{z}}$ can be factorized into a completely antisymmetric color wave function, $\Gamma$, and a symmetric spin-flavor-momentum state, $\Phi_{s f}$, as follows:

$$
\Psi_{r S_{z}}=\Phi_{s f, S_{z}}\left(\vec{k}_{1}\left\{m_{1}, \mathcal{Q}\right\} ; \vec{k}_{3}\left\{m_{3}, \mathcal{F}_{3}\right\} ;-\vec{k}_{1}-\vec{k}_{3},\left\{m_{n}, \mathcal{F}_{n}\right\}\right) \Gamma\left(i, c_{3}, c_{n}\right)
$$

The matrix element of the color operator in Eq. (15) can be therefore immediately evaluated:

$$
\sum_{c_{3}, c_{4}, i, j} \Gamma^{\dagger}\left(i, c_{3}, c_{n}\right) T_{i j}^{a} T_{c_{3} c_{4}}^{a} \Gamma\left(j, c_{4}, c_{n}\right)=-\frac{2}{3}
$$

which is the well-known result for the exchange of one gluon between quarks in a color singlet 3-quark state [40]. Besides, as a consequence of the symmetry of the state $\Phi_{s f}$, one can perform the calculation assuming that the interacting quark is the one labeled " 1 ", so that, after the evaluation of the summation on the flavors $\mathcal{F}_{3}, \mathcal{M}^{\alpha}$ can be written, for the $u$ and $d$ flavors, as follows:

$$
\begin{aligned}
\mathcal{M}^{u(d)} & =\left(-\frac{2}{3}\right) \cdot 3 \cdot \frac{1}{2} \sum_{S_{z}=-1,1} \sum_{m_{1}, m_{2}, m_{3}, m_{4}} \Phi_{s f, S_{z}}^{\dagger}\left(\vec{k}_{1}, m_{1} ; \vec{k}_{3}, m_{3} ;-\vec{k}_{1}-\vec{k}_{3}, m_{n}\right) \\
& \times \frac{1 \pm \tau_{3}(1)}{2} V_{\mathrm{NR}}^{h}\left(\vec{k}_{1}, \vec{k}_{3}, \vec{q}\right) \\
& \times \Phi_{s f, S_{z}}\left(\vec{k}_{1}+\vec{q}, m_{2} ; \vec{k}_{3}-\vec{q}, m_{4} ;-\vec{k}_{1}-\vec{k}_{3}, m_{n}\right),
\end{aligned}
$$


where the helicity $m_{n}$ of the spectator quark is determined by $m_{1}, m_{3}$ and $S_{z}$ due to angular momentum conservation. Some remarks concerning the interaction, Eq. (16), are in order. In general, the interaction can be separated according to the possible helicity combinations of the interacting quarks in the initial and final state. Besides, since the wave functions to be used are NR, a NR reduction of the interaction has to be obtained. This NR reduction is given by the $V_{\mathrm{NR}}^{h}\left(\vec{k}_{1}, \vec{k}_{3}, \vec{q}\right)$ expression in Eq. (19). In order to obtain it, we follow the procedure developed for the Sivers function in Ref. [4], using therefore the definitions of free four-spinors in Eq. (16) and performing a NR expansion as it is commonly done in nuclear physics. At $O\left(k^{2} / M^{2}\right)$ one obtains

$$
V_{\mathrm{NR}}^{h}\left(\vec{k}_{1}, \vec{k}_{3}, \vec{q}\right)=V_{\mathrm{NR}}^{n o-f l i p}\left(\vec{k}_{1}, \vec{k}_{3}, \vec{q}\right)+V_{\mathrm{NR}}^{\text {double-flip }}\left(\vec{k}_{1}, \vec{k}_{3}, \vec{q}\right)
$$

i.e., the relevant processes for the evaluation of $h_{1}^{\perp}$ are the ones where either the interacting and active quarks do not flip their helicities, or they flip them both. The expressions for the $V_{\mathrm{NR}}^{\text {no-flip }}$ and $V_{\mathrm{NR}}^{\text {double-flip }}$ potentials are given in the Appendix. As explained in Ref. [4] in the case of the Sivers function, we reiterate that, in this approach, it is the interference of the upper and lower components of the four-spinors of the free quark states which leads to a non-vanishing $h_{1}^{\perp}$. Effectively, these interference terms in the interaction are the ones that, in the MIT bag model previously described, arise due to the wave function. Terms of higher order would correspond to those arising, in the MIT bag model calculation, from the product of the lower components of the quark wave function, a contribution which has been found to be negligible. The results of the relativistic calculation justifies therefore the NR reduction of the interaction in the CQM calculation.

Eq. (14), with $\mathcal{M}^{u(d)}$ given by Eq. (19), provides a suitable formula to evaluate $h_{1}^{\perp}$ using the proton state in momentum space, $\Phi_{s f}$, described in a CQM. We will here restrict our calculation to a Harmonic Oscillator potential model with pure SU(6) symmetry for the proton. The choice of a $\mathrm{SU}(6)$ wave function is motivated to render the comparison with the previous calculation, performed in the MIT bag model with SU(6) symmetry, more plausible. It is worth recalling that, as it has been stressed in Ref. [4], the breaking of SU(6) in the CQM of Isgur and Karl [41] does not change essentially the results of the calculation. This has been obtained also in the evaluation of standard PDs or GPDs in quark models (see, i.e. [42]): the essential features of a CQM calculation are obtained in a pure SU(6) framework. The formal expression of the proton state can be given in terms of the sets of 
conjugated intrinsic coordinates given in the Appendix. It reads

$$
\left.\left.\right|^{2} S_{1 / 2}\right\rangle_{S}=\frac{e^{-\left(k_{\rho}^{2}+k_{\lambda}^{2}\right) / \alpha^{2}}}{\pi^{3 / 2} \alpha^{3}}|\chi\rangle_{S},
$$

where the spectroscopic notation $\left.\left.\right|^{2 S+1} X_{J}\right\rangle_{t}$ with $t=A, M, S$ being the symmetry type, has been used, and $|\chi\rangle_{S}$ is the standard $\mathrm{SU}(6)$ vector describing the spin-flavor structure of the proton. The parameter $\alpha^{2}=m \omega$ of the H.O. potential is fixed to the value $1.35 \mathrm{fm}^{-2}$, in order to reproduce the slope of the proton charge form factor at zero momentum transfer [40].

We have now all the necessary ingredients to write the final expression for $h_{1}^{\perp u(d)}$, which reads

$$
\begin{aligned}
& h_{1}^{\perp u(d)}\left(x, k_{T}\right)=g^{2} \frac{M^{2}}{k_{x}}\left(\frac{3}{2}\right)^{3 / 2} \frac{1}{2 \pi^{3 / 2} \alpha^{3}} \int \frac{d^{2} \vec{q}_{T}}{(2 \pi)^{2}} \sqrt{\frac{3}{2}} \frac{\sqrt{2} k_{\lambda}^{0}}{\left|k_{\lambda}^{0}-k_{\lambda}^{z}\right|} \frac{1}{q^{2}} e^{-\frac{1}{\alpha^{2}}\left[k_{\lambda}^{2}+\frac{7}{8} q_{T}^{2}-\sqrt{\frac{3}{2}} \vec{q} \cdot \vec{k}_{\lambda}\right]} \\
& {\left[1(0) B+\frac{1}{3}\left(\frac{2}{3}\right)\left\{A+\frac{q^{2} q^{x}}{48 m^{2}}\right\}\right] }
\end{aligned}
$$

with $k_{\lambda}^{0}=\sqrt{m^{2}+k_{\lambda}^{2}}$, and

$$
\begin{aligned}
& \vec{k}_{\lambda}=\sqrt{\frac{3}{2}}(\vec{q}-\vec{k}), \quad k_{\lambda}^{z}=\frac{\frac{3}{2} m^{2}+\vec{k}_{\lambda T}^{2}-3 x^{2} P^{+2}}{2 \sqrt{3} P^{+} x} \\
& k_{\lambda}^{2}=k_{\lambda}^{z 2}+\vec{k}_{\lambda T}^{2}
\end{aligned}
$$

The explicit expressions for the functions $A, B$ are given in the Appendix.

\section{RESULTS AND DISCUSSION}

To evaluate numerically Eqs. (13) and (22), the strong coupling constant $g$, and therefore $\alpha_{s}\left(Q^{2}\right)$, has to be fixed. Here, the model-independent prescription introduced for calculations of PDs in quark models (see, i.e., Ref. [42]) will be used. It consists in fixing the momentum scale of the model, the so-called hadronic scale $\mu_{0}^{2}$, according to the amount of momentum carried by the valence quarks. In the present approach, for both the MIT bag model and the CQM, only valence quarks contribute. The assumption that all the gluons and sea pairs in the proton are produced perturbatively, according to NLO evolution equations, yields $\mu_{0}^{2} \simeq 0.1 \mathrm{GeV}^{2}$, if $\Lambda_{Q C D}^{N L O} \simeq 0.24 \mathrm{GeV}$. Therefore, one has $\alpha_{s}\left(\mu_{0}^{2}\right) /(4 \pi) \simeq 0.13$ [42]. This is obtained imposing that $\simeq 55 \%$ of the momentum is carried by the valence quarks at a scale of $0.34 \mathrm{GeV}^{2}$, as in typical low-energy parameterizations (see, i.e., Ref. [44]). 
The first moments of the Sivers and Boer-Mulders functions, i.e. the quantities

$$
q^{(1)}(x)=\int d^{2} \vec{k}_{T} \frac{k_{T}^{2}}{2 M^{2}} q\left(x, k_{T}\right),
$$

where $q=h_{1}^{\perp \mathcal{Q}}$, or $f_{1 T}^{\perp \mathcal{Q}}$, are depicted in Figs. 2-5. The results for the calculation in the MIT bag model as well as in the CQM are represented for both functions on Fig. 2. As already mentioned in Refs. [4, 5], the signs of the Sivers functions for the $u$ and $d$ flavors, negative and positive respectively, are in agreement with both the theoretical and experimental knowledge. Concerning the Boer-Mulders function, no data are available but following the pattern predicted by quark helicity-flip IPD GPDs [30, 31], the same sign for the $u$ and $d$ flavors is expected. This is found in the present approach, confirming the results of previous estimates, such as the first MIT bag model calculation [33], the quark-diquark model [28], a large $N_{c}$ analysis [34] and a phenomenological parameterization based on the quark-diquark picture [35]. This can be clearly seen in the lower panels of Fig. 2. The good performances of the approach, established in the calculation of the Sivers function, are therefore confirmed here for the Boer-Mulders function.

For the sake of clarity, the same results are rearranged in Fig. 3, such that one can compare, in both models, the Sivers with the Boer-Mulders functions. It turns out that the shape of both functions is similar in the CQM and in the MIT bag model. In particular, the absolute value of $h_{1}^{\perp}$ turns out to be a little bigger than that of $f_{1 T}^{\perp}$. Also the size is not too different, since it crucially depends on the scale of the model, which fixes the strong coupling constant $g$ appearing in Eqs. (13) and (22). A small variation of $g$ affects dramatically the results. We have decided to fix the hadronic scale as explained at the beginning of this section, i.e. in a model independent way, obtaining $\alpha_{s}\left(\mu_{0}^{2}\right) /(4 \pi) \simeq 0.13$. One could have instead taken the initial scales corresponding to the ones fixed for the two different models by the Authors proposing them, i.e. $\alpha_{s}\left(\mu_{0}^{2}\right) /(4 \pi) \simeq 0.11$ for the CQM [40] and $\alpha_{s}\left(\mu_{0}^{2}\right) /(4 \pi) \simeq 0.18$ for the MIT bag [43], respectively. The sizes of the results in the two different models would have come out more similar. Since there are no precise data available, a finer tuning of these parameters is not relevant at this moment.

In Figs. 4 and 5, a microscopic analysis of the contributions of different helicity-flips of the quark interacting with the virtual photon and of the active recoiling one is presented, for $f_{1 T}^{\perp}$ and $h_{1}^{\perp}$, respectively.

In the case of the Sivers function, the helicity-flip can occur either for the quark interacting 
with the virtual photon, or for the active recoiling one [4, 5]. The latter contribution has been disregarded in Ref. [33], while it is seen in Fig. 4 that it is very important. We recall that, once such a term is taken properly into account, the Burkardt Sum Rule is fulfilled to a large extent [5]. A similar problem occurs also for the calculation of $h_{1}^{\perp}$. In the previous section, we have shown that, in the case of this function, contributions are found if there is no helicity-flip for the two active quarks, or if they both flip their helicities (cf. Eq. (13) and (20) $)$. The latter contribution has been once more disregarded in Ref. [33], while it is seen in Fig. 5 that it is sizeable. Moreover, if the two contributions are disregraded, $f_{1 T}^{\perp}$ for a given flavor turns out to be proportional to $h_{1}^{\perp}$ for the same flavor. Besides, $f_{1 T}^{\perp}\left(h_{1}^{\perp}\right)$ for a flavor turns out to be proportional to $f_{1 T}^{\perp}\left(h_{1}^{\perp}\right)$ for the other flavor.

These proportionalities, are not found once the proper helicity-flip contributions are taken into account. Indeed, the reincorporated term is subtractive in the case of the Sivers function, while it is additive in the case of the Boer-Mulders function. This implies that the two T-odd functions cannot be proportional. In any case, the proportionality found in Ref. [33] has no physical motivation, as, in order to calculate the two T-odd functions, one is using a twobody operator associated with FSI and therefore one should not expect a proportionality between the $u$ and $d$ results. On the contrary, in the calculation of conventional PDs, in any $\mathrm{SU}(6)$ model calculation, the used operators are of one-body type and therefore the results turn out to be proportional [39].

Another interesting byproduct of the present results is that there is basically no qualitative difference between the results in the two models, despite the fact that one of them is NR. As already said in the previous section, this has to do with the fact that the terms which encode the most relativistic contribution arising in the MIT bag model turn out to be a few orders of magnitude smaller than the dominant ones. This is seen in Eqs. (A8) and (A14) in the Appendix at the formal level. This gives us more confidence in the order $O\left(k^{2} / m^{2}\right)$ of the NR expansion used.

Let us now compare our results with the other microscopic calculation of the Boer-Mulders function, the one in the quark-diquark model [28]. We get the same, negative, sign for the $u$ and $d$ flavors, which has been proven to be related to the model independent features of IPD GPDS [30]. On the other hand, the relative size of the $u$ and $d$ flavors turn out to be different (at variance with [28], we have the result for $u$ a bit larger than for $d$ ). Anyway, as far as we understand, a model parameter in Ref. [28] can change such a relative size, 
making this comparison not conclusive. What makes our approach unique, transparent and instructive, is that a microscopic analysis of the helicity-flip of the different quarks involved is not possible in two-body models, such as the quark-diquark model. We have seen instead that one can learn several interesting aspects of the problem by performing this helicity-flip analysis at the single quark level.

One relevant missing part in our analysis is the evaluation of the pQCD evolution of the results from the scale of the model to the experimental one. Unfortunately, to our knowledge, the anomalous dimensions of the relevant operators have not been calculated yet and no workable evolution equations have been provided. Some crucial steps towards the solution of this problem have been done [45, 46, 47, 48, 49, 50], and they will be considered in future work.

Other natural developments of this calculation are the analysis in a relativistic three-body model, as well as the connection of T-odd TMDs with the corresponding IPD GPDs.

\section{CONCLUSIONS}

We have applied a formalism, developed previously to calculate the Sivers function, to the calculation of the Boer-Mulders function with the aim of understanding the microscopic mechanisms associated with the transverse polarization of the nucleon. The interest of the calculation lies in the fact that, despite the difficult experimental extraction of this function, ongoing experiments will produce data. We have applied the formalism to two very different models of nucleon structure: a non relativistic constituent quark model and a relativistic MIT bag model, both of which are able to interpret well the static properties of the proton. Our calculation is complete in the sense that it takes into account all possible contributions, i.e. not only the helicity non-flip contribution, but also the helicity double flip one.

The calculated moment of the Boer-Mulders function has the same negative sign for the $u$ and $d$ flavors confirming the results of previous predictions and estimates. This nice outcome also happened in our previous calculation of the Sivers function using the same formalism, where our results satisfied the Burkardt Sum Rule and followed the general trend of previous correct estimates. The shapes of both functions, Sivers and Boer-Mulders, are similar in the CQM and the MIT bag model. This is a consequence of the fact that the terms carrying the most relativistc contribution in the bag model are a few orders of magnitude smaller thean 
the main contributions. It should be stressed that, the additional terms found in both of these functions in the MIT bag model calculations, are crucial to establish this behavior and also to satisfy fundamental relations like the Burkardt Sum Rule.

To conclude, we have developed a formalism that allows a straightforward evaluation of all kinds of parton distributions, which can be easily applied to different models of hadron structure and, in particular, to two of the most succesful ones. Moreover the results obtained are quite model independent in structure, and are in agreement with fundamental principles and predictions. Our analysis has the advantage, with respect to other analyses in other formalisms, that it shows the microscopic separation of the different helicity-flip contributions for the different quarks involved in the process and, in so doing, is able to understand the microscopic structure of the transverse polarization up to evolution effects.

\section{APPENDIX A: SOME DETAILS OF THE RESULTS OF THE CALCULATION}

Some details are listed below.

\section{MIT bag model calculation}

The matrix elements Eq. (12) are listed below, for $\mathcal{Q}=u$ and $S_{z}=1(-1)$,

$$
\begin{gathered}
C_{u S_{z}=1(-1)}^{++,++}=-\frac{5}{9}(0), \quad C_{u S_{z}=1(-1)}^{++,--}=-\frac{5}{9}\left(-\frac{2}{9}\right), \quad C_{u S_{z}=1(-1)}^{--,++}=-\frac{2}{9}\left(-\frac{5}{9}\right), \\
C_{u S_{z}=1(-1)}^{--,--}=0\left(-\frac{5}{9}\right) C_{u S_{z}=1(-1)}^{+-,+-}=0(0), \quad C_{u S_{z}=1(-1)}^{+-,-+}=\frac{1}{9}\left(\frac{1}{9}\right), \\
C_{u S_{z}=1(-1)}^{-+,+-}=\frac{1}{9}\left(\frac{1}{9}\right), \quad C_{u S_{z}=1(-1)}^{-+,-+}=0(0),
\end{gathered}
$$

and for $\mathcal{Q}=d$ and $S_{z}=1(-1)$,

$$
\begin{gathered}
C_{d S_{z}=1(-1)}^{++,++}=-\frac{1}{9}(0), \quad C_{d S_{z}=1(-1)}^{++,--}=-\frac{1}{9}\left(-\frac{4}{9}\right), \quad C_{d S_{z}=1(-1)}^{--,++}=-\frac{4}{9}\left(-\frac{1}{9}\right), \\
C_{d S_{z}=1(-1)}^{--,--}=0\left(-\frac{1}{9}\right) \quad C_{d S_{z}=1(-1)}^{+-,+-}=0(0), \quad C_{d S_{z}=1(-1)}^{+-,-+}=\frac{2}{9}\left(\frac{2}{9}\right),
\end{gathered}
$$




$$
C_{d S_{z}=1(-1)}^{-+,+-}=\frac{2}{9}\left(\frac{2}{9}\right), \quad C_{d S_{z}=1(-1)}^{-+,-+}=0(0) \quad .
$$

The functions entering Eq. (13) are listed below:

$$
\begin{aligned}
F_{1} & =\int d^{3} \vec{k}_{3}\left\{t_{0}\left(k_{3}\right) t_{0}\left(k_{3}^{\prime}\right)+k_{3}^{z} \frac{t_{1}\left(k_{3}\right)}{k_{3}} t_{0}\left(k_{3}^{\prime}\right)+k_{3}^{z} \frac{t_{1}\left(k_{3}^{\prime}\right)}{k_{3}^{\prime}} t_{0}\left(k_{3}\right)\right. \\
& \left.+\left(k_{3}^{2}-\vec{k}_{3} \cdot \vec{q}_{T}\right) \frac{t_{1}\left(k_{3}\right)}{k_{3}} \frac{t_{1}\left(k_{3}^{\prime}\right)}{k_{3}^{\prime}}\right\} \\
F_{2} & =\int d^{3} \vec{k}_{3}\left\{\left(\vec{q}_{T} \times \vec{k}_{3}\right)^{z} \frac{t_{1}\left(k_{3}\right)}{k_{3}} \frac{t_{1}\left(k_{3}^{\prime}\right)}{k_{3}^{\prime}}\right\} \\
H_{1} & =\int d^{3} \vec{k}_{3}\left\{k_{3}^{x} \frac{t_{1}\left(k_{3}\right)}{k_{3}} t_{0}\left(k_{3}^{\prime}\right)-\left(k_{3}^{x}-q^{x}\right) \frac{t_{1}\left(k_{3}^{\prime}\right)}{k_{3}^{\prime}} t_{0}\left(k_{3}\right)-\left(\vec{q}_{T} \times \vec{k}_{3}\right)^{y} \frac{t_{1}\left(k_{3}\right)}{k_{3}} \frac{t_{1}\left(k_{3}^{\prime}\right)}{k_{3}^{\prime}}\right\} \\
H_{2} & =-\int d^{3} \vec{k}_{3}\left\{k_{3}^{y} \frac{t_{1}\left(k_{3}\right)}{k_{3}} t_{0}\left(k_{3}^{\prime}\right)-\left(k_{3}^{y}-q^{y}\right) \frac{t_{1}\left(k_{3}^{\prime}\right)}{k_{3}^{\prime}} t_{0}\left(k_{3}\right)+\left(\vec{q}_{T} \times \vec{k}_{3}\right)^{x} \frac{t_{1}\left(k_{3}\right)}{k_{3}} \frac{t_{1}\left(k_{3}^{\prime}\right)}{k_{3}^{\prime}}\right\},
\end{aligned}
$$

and

$$
\begin{aligned}
I_{1} & =k^{x} \frac{t_{1}(k)}{k} t_{0}\left(k^{\prime}\right)-\left(k^{x}-q^{x}\right) \frac{t_{1}\left(k^{\prime}\right)}{k^{\prime}} t_{0}(k)-\left(k^{\prime x} k^{z}-k^{\prime} z k^{x}\right) \frac{t_{1}(k)}{k} \frac{t_{1}\left(k^{\prime}\right)}{k^{\prime}}, \\
I_{2} & =k^{y} \frac{t_{1}(k)}{k} t_{0}\left(k^{\prime}\right)+\left(k^{y}-q^{y}\right) \frac{t_{1}\left(k^{\prime}\right)}{k^{\prime}} t_{0}(k)+\left(k^{\prime y} k^{z}+k^{\prime z} k^{y}\right) \frac{t_{1}(k)}{k} \frac{t_{1}\left(k^{\prime}\right)}{k^{\prime}}, \\
J_{1} & =t_{0}(k) t_{0}\left(k^{\prime}\right)+k^{z} \frac{t_{1}(k)}{k} t_{0}\left(k^{\prime}\right)+k^{z} \frac{t_{1}\left(k^{\prime}\right)}{k^{\prime}} t_{0}(k) \\
& +\left(k^{x 2}-q^{x} k^{x}-k^{y 2}+q^{y} k^{y}+k^{z 2}\right) \frac{t_{1}(k)}{k} \frac{t_{1}\left(k^{\prime}\right)}{k^{\prime}}, \\
J_{2} & =-\left(2 k^{x} k^{y}-q^{x} k^{y}-q^{y} k^{x}\right) \frac{t_{1}(k)}{k} \frac{t_{1}\left(k^{\prime}\right)}{k^{\prime}},
\end{aligned}
$$

with $k=|\vec{k}|$ and $\vec{k}^{\prime}=\vec{k}-\vec{q}_{T}$.

The constant $C$ entering Eq. (13) is:

$$
C=\sqrt{\frac{2}{9}} \frac{C^{\prime}}{(2 \pi)^{3}},
$$

with

$$
C^{\prime}=4 \pi N^{2} R_{0}^{6}=\frac{16 \omega^{4}}{\pi^{2} j_{0}^{2}(\omega)(\omega-1)} \frac{(2 \pi)^{3}}{M_{P}^{3}},
$$

determined by the MIT bag model parameters. 


\section{CQM calculation}

The potentials corresponding to gluon exchanges without quark helicity flips or with double quark helicity flips, Eq. (20), are listed below:

$$
\begin{aligned}
& V^{n o-f l i p}\left(\vec{k}_{3}, \vec{k}_{1}, \vec{q}\right)=\frac{1}{2 q^{2}}\left\{-i\left(1+\frac{k_{1}^{z}}{m}+\frac{\vec{q} \cdot \vec{k}_{1}}{4 m^{2}}\right)\left(\frac{q^{x}}{2 m}+\frac{q^{x} k_{3}^{z}}{4 m^{2}}\right)\right. \\
& +\left(1+\frac{k_{1}^{z}}{m}+\frac{\vec{q} \cdot \vec{k}_{1}}{4 m^{2}}\right)\left(-\frac{k_{3}^{y}}{m}+\frac{q^{y}}{2 m}+\frac{q^{y} k_{3}^{z}}{4 m^{2}}\right) \sigma_{3}^{z} \\
& +\frac{1}{4 m^{2}}\left(k_{1}^{x} q^{y}-q^{x} k_{1}^{y}\right)\left(\frac{q^{x}}{2 m}+\frac{q^{x} k_{3}^{z}}{4 m^{2}}\right) \sigma_{1}^{z} \\
& \left.+\frac{i}{4 m^{2}}\left(k_{1}^{x} q^{y}-q^{x} k_{1}^{y}\right)\left(-\frac{k_{3}^{y}}{m}+\frac{q^{y}}{2 m}+\frac{q^{y} k_{3}^{z}}{4 m^{2}}\right) \sigma_{3}^{z} \sigma_{1}^{z}\right\} . \\
& V^{\text {double-flip }}\left(\vec{k}_{3}, \vec{k}_{1}, \vec{q}\right)=\frac{1}{2 q^{2}}\left\{-\frac{i}{4 m^{2}}\left(\frac{q^{y}}{2 m}+\frac{q^{y} k_{1}^{z}}{4 m^{2}}\right)\left(k_{3}^{x} q^{y}+k_{3}^{y} q^{x}\right) \sigma_{3}^{x} \sigma_{1}^{x}\right. \\
& -i\left(\frac{q^{y}}{2 m}+\frac{q^{y} k_{1}^{z}}{4 m^{2}}\right)\left(1+\frac{k_{3}^{z}}{m}+\frac{q^{y} k_{3}^{y}-q^{x} k_{3}^{x}}{4 m^{2}}\right) \sigma_{3}^{y} \sigma_{1}^{x} \\
& +\frac{i}{4 m^{2}}\left(\frac{q^{x}}{2 m}+\frac{q^{x} k_{1}^{z}}{4 m^{2}}\right)\left(k_{3}^{x} q^{y}+k_{3}^{y} q^{x}\right) \sigma_{3}^{x} \sigma_{1}^{y} \\
& \left.+i\left(\frac{q^{x}}{2 m}+\frac{q^{x} k_{1}^{z}}{4 m^{2}}\right)\left(1+\frac{k_{3}^{z}}{m}+\frac{q^{y} k_{3}^{y}-q^{x} k_{3}^{x}}{4 m^{2}}\right) \sigma_{3}^{y} \sigma_{1}^{y}\right\}
\end{aligned}
$$

The set of conjugated intrinsic coordinates used in Eq. (21) is

$$
\begin{gathered}
\vec{R}=\frac{1}{\sqrt{3}}\left(\overrightarrow{r_{1}}+\overrightarrow{r_{2}}+\overrightarrow{r_{3}}\right) \leftrightarrow \vec{K}=\frac{1}{\sqrt{3}}\left(\overrightarrow{k_{1}}+\overrightarrow{k_{2}}+\overrightarrow{k_{3}}\right), \\
\vec{\rho}=\frac{1}{\sqrt{2}}\left(\overrightarrow{r_{3}}-\overrightarrow{r_{2}}\right) \leftrightarrow \overrightarrow{k_{\rho}}=\frac{1}{\sqrt{2}}\left(\overrightarrow{k_{3}}-\overrightarrow{k_{2}}\right), \\
\vec{\lambda}=\frac{1}{\sqrt{6}}\left(\overrightarrow{r_{2}}+\overrightarrow{r_{3}}-2 \overrightarrow{r_{1}}\right) \leftrightarrow \overrightarrow{k_{\lambda}}=\frac{1}{\sqrt{6}}\left(\overrightarrow{k_{2}}+\overrightarrow{k_{3}}-2 \overrightarrow{k_{1}}\right) .
\end{gathered}
$$

The $B$ and $A$ functions appearing in Eq. (22) are

$$
\begin{aligned}
& B=-\frac{q^{x}}{2 m}\left(1+\frac{k_{\rho}^{z}}{2 \sqrt{2}}-\frac{3}{\sqrt{6}} \frac{\bar{k}_{\lambda}^{z}}{2 m}-\frac{q_{x}^{2}+q_{y}^{2}-q_{x} k_{x}-q_{y} k_{y}}{4 m^{2}}\right) \\
& A=-\frac{5}{6} \frac{q_{x}}{2 m}+\frac{3}{\sqrt{6}} \frac{\bar{k}_{\lambda}^{z} q_{x}}{4 m^{2}}+\frac{1}{24 m^{3}}\left(4 q_{x}^{3}-4 q_{x}^{2} k_{x}+4 q_{x} q_{y}^{2}-q_{x} q_{y}^{2}-q_{x} q_{y} k_{y}-3 k_{x} q_{y}^{2}\right)
\end{aligned}
$$




\section{ACKNOWLEDGMENTS}

We are grateful to S. Noguera and G. Goldstein for fruitful discussions, and J.C. Peng and A. Prokudin who suggested us to start this investigation. S.S. thanks the Department of Theoretical Physics of the Valencia University for warm hospitality; A.C. and V.V. thank the Department of Physics of the Perugia University for warm hospitality. This work is supported in part by the INFN-MEC agreement FPA2008-03646-E/INFN; by HadronPhysics2, a FP7-Integrating Activities and Infrastructure Program of the European Commission, under Grant 227431; by the MICINN (Spain) grant FPA2007-65748-C02-01 and the grant AP2005-5331.

[1] V. Barone, A. Drago and P. G. Ratcliffe, Phys. Rept. 359 (2002) 1 arXiv:hep-ph/0104283.

[2] Proceedings of "Transversity 2008”, May 2008, Ferrara, Italy, 2009 in press.

[3] D. Boer and P. J. Mulders, Phys. Rev. D 57 (1998) 5780 arXiv:hep-ph/9711485.

[4] A. Courtoy, F. Fratini, S. Scopetta and V. Vento, Phys. Rev. D 78 (2008) 034002 arXiv:0801.4347 [hep-ph]].

[5] A. Courtoy, S. Scopetta and V. Vento, Phys. Rev. D 79, 074001 (2009) arXiv:0811.1191 [hep-ph]].

[6] D. W. Sivers, Phys. Rev. D 41, 83 (1990), Phys. Rev. D 43, 261 (1991).

[7] M. Burkardt, Phys. Rev. D 69 (2004) 091501; Phys. Rev. D 69 (2004) 057501.

[8] M. Anselmino, M. Boglione, U. D'Alesio, A. Kotzinian, F. Murgia and A. Prokudin, Phys. Rev. D 71, 074006 (2005) arXiv:hep-ph/0501196,M. Anselmino, M. Boglione, U. D'Alesio, A. Kotzinian, F. Murgia and A. Prokudin, Phys. Rev. D 72, 094007 (2005) [Erratum-ibid. D 72, 099903 (2005)] arXiv:hep-ph/0507181]. A. V. Efremov, K. Goeke, S. Menzel, A. Metz and P. Schweitzer, Phys. Lett. B 612, 233 (2005) arXiv:hep-ph/0412353, J. C. Collins, A. V. Efremov, K. Goeke, S. Menzel, A. Metz and P. Schweitzer, Phys. Rev. D 73, 014021 (2006) arXiv:hep-ph/0509076 W. Vogelsang and F. Yuan, Phys. Rev. D 72, 054028 (2005) arXiv:hep-ph/0507266.

[9] S. J. Brodsky, D. S. Hwang and I. Schmidt, Phys. Lett. B 530, 99 (2002) arXiv:hep-ph/0201296. 
[10] S. J. Brodsky, P. Hoyer, N. Marchal, S. Peigne and F. Sannino, Phys. Rev. D 65, 114025 (2002) arXiv:hep-ph/0104291.

[11] J. C. Collins, Phys. Lett. B 536, 43 (2002) arXiv:hep-ph/0204004.

[12] X. d. Ji and F. Yuan, Phys. Lett. B 543, 66 (2002) arXiv:hep-ph/0206057].

[13] A. V. Belitsky, X. Ji and F. Yuan, Nucl. Phys. B 656, 165 (2003) arXiv:hep-ph/0208038.

[14] M. Burkardt, Nucl. Phys. A 735, 185 (2004) arXiv:hep-ph/0302144); Phys. Rev. D 66, 114005 (2002) arXiv:hep-ph/0209179; M. Burkardt and D. S. Hwang, Phys. Rev. D 69, 074032 (2004) arXiv:hep-ph/0309072.

[15] M. Burkardt, Int. J. Mod. Phys. A 18, 173 (2003) arXiv:hep-ph/0207047].

[16] S. Meissner, A. Metz and K. Goeke, Phys. Rev. D 76, 034002 (2007) arXiv:hep-ph/0703176].

[17] G. Schnell, Talk at the CLAS12 European Workshop February 25-28, 2009- Genova, Italy

[18] A. Bressan and f. t. C. Collaboration, arXiv:0907.5511 [hep-ex].

[19] F. Giordano and R. Lamb [On behalf of the HERMES Collaboration], section at AIP Conf. Proc. 1149 (2009) 423 arXiv:0901.2438 [hep-ex]].

[20] V. Barone, A. Prokudin and B. Q. Ma, Phys. Rev. D 78 (2008) 045022 arXiv:0804.3024 [hep-ph]].

[21] G. Bunce, N. Saito, J. Soffer and W. Vogelsang, Ann. Rev. Nucl. Part. Sci. 50 (2000) 525 arXiv:hep-ph/0007218.

[22] L.Y. Zhu et al., [FNAL-E866/NuSea], Phys. Rev. Lett. 99, 082301 (2007); Phys. Rev. Lett. 102, 182001, (2009); J.C. Peng, Proceedings of the $6^{\text {th }}$ International Conference on Perspectives in Hadronic Physics, Trieste, Italy, May 12-16 2008, AIP Conference Proceedings, vol. 1056, 452 (2008).

[23] V. Barone et al. [PAX Collaboration], arXiv:hep-ex/0505054.

[24] B. Zhang, Z. Lu, B. Q. Ma and I. Schmidt, Phys. Rev. D 77 (2008) 054011 arXiv:0803.1692 [hep-ph]].

[25] J. C. Collins, Nucl. Phys. B 396, 161 (1993) arXiv:hep-ph/9208213.

[26] D. Boer, Phys. Rev. D 60 (1999) 014012 arXiv:hep-ph/9902255].

[27] A. Bacchetta, A. Schaefer and J. J. Yang, Phys. Lett. B 578, 109 (2004) arXiv:hep-ph/0309246.

[28] L.P. Gamberg. G.R. Goldstein, and M. Schlegel, Phys. Rev. D 77, 094016 (2008).

[29] L.P. Gamberg. G.R. Goldstein, and K.A. Oganessyan, Phys. Rev. D 67, 071504 (2003); Phys. 
Rev. D 68, 051501 (2003).

[30] M. Burkardt and B. Hannafious, Phys. Lett. B 658, 130 (2008).

[31] B. Pasquini and S. Boffi, Phys. Lett. B 653, 23 (2007); e-Print: arXiv:0705.4345 [hep-ph]

[32] M. Gockeler et al. [QCDSF Collaboration and UKQCD Collaboration], Phys. Rev. Lett. 98 (2007) 222001 arXiv:hep-lat/0612032.

[33] F. Yuan, Phys. Lett. B 575, 45 (2003) arXiv:hep-ph/0308157].

[34] P. V. Pobylitsa, arXiv:hep-ph/0301236.

[35] A. Bacchetta, F. Conti, and M. Radici, Phys. Rev. D 78, 074010 (2008).

[36] A. Bacchetta, U. D'Alesio, M. Diehl and C. A. Miller, Phys. Rev. D 70, 117504 (2004) arXiv:hep-ph/0410050.

[37] X. d. Ji, J. p. Ma and F. Yuan, Phys. Rev. D 71 (2005) 034005 arXiv:hep-ph/0404183.

[38] J. C. Collins and A. Metz, Phys. Rev. Lett. 93, 252001 (2004) arXiv:hep-ph/0408249.

[39] R. L. Jaffe, Phys. Rev. D 11 (1975) 1953.

[40] M. M. Giannini, Rept. Prog. Phys. 54, 453 (1990).

[41] N. Isgur and G. Karl, Phys. Rev. D 18, 4187 (1978); Phys. Rev. D 19, 2653 (1979) [Erratumibid. D 23, 817 (1981)].

[42] S. Scopetta and V. Vento, Phys. Lett. B 424, 25 (1998) arXiv:hep-ph/9706413. S. Scopetta and V. Vento, Phys. Lett. B 460, 8 (1999) [Erratum-ibid. B 474, 235 (2000)] arXiv:hep-ph/9901324. M. Traini, A. Mair, A. Zambarda and V. Vento, Nucl. Phys. A 614, 472 (1997). S. Scopetta and V. Vento, Eur. Phys. J. A 16, 527 (2003) arXiv:hep-ph/0201265.

[43] A.W. Thomas and W. Weise, "The structure of the nucleon", Wiley-VCH, Berlin, 2001.

[44] M. Gluck, E. Reya and A. Vogt, Eur. Phys. J. C 5, 461 (1998) arXiv:hep-ph/9806404.

[45] F. A. Ceccopieri and L. Trentadue, Phys. Lett. B 636, 310 (2006) arXiv:hep-ph/0512372; arXiv:0706.4242 [hep-ph].

[46] I. O. Cherednikov and N. G. Stefanis, Nucl. Phys. B 802 (2008) 146 [arXiv:0802.2821 [hep-ph]].

[47] W. Vogelsang and F. Yuan, Phys. Rev. D 79, 094010 (2009) arXiv:0904.0410 [hep-ph]].

[48] Z-B. Kang, J-W. Qiu, Phys. Rev. D79, 016003, (2009).

[49] J. Zhou, F. Yuan, Z-T Liang, Phys. Rev. D79, 114022, (2009).

[50] V.M. Braun, A.N. Manashov, B. Pirnay, e-Print: arXiv:0909.3410 [hep-ph].

[51] D. Binosi and L. Theussl, Comput. Phys. Commun. 161 (2004) 76 arXiv:hep-ph/0309015]. 


\section{FIGURE CAPTIONS}

Fig. 1: The contributions to the Sivers and Boer-Mulders functions in the present approach. The graph has been drawn using JaxoDraw [51].

Fig. 2: Upper (lower) panel, left: the first moment of the Sivers (Boer-Mulders) function, Eq. (24), for the $u$ flavor, in the NR (full) and MIT bag models (dashed); right: the same for the $d$ flavor.

Fig. 3: Left panel: the first moment for the Sivers and Boer-Mulders functions in the NR model. Dashed curve: the Sivers function for $d$; dot-dashed curve: the Sivers function for $u$; long dashed curve: the Boer-Mulders function for $d$; full curve: the Boer-Mulders function for $u$. Right panel: the same in the MIT Bag model.

Fig. 4: Comparison between the results for the first moment of the Sivers function in the NR model and in the MIT bag model. Left panel: results in the NR model. Full (Long dashed) curve: full result for the flavor $u(d)$; dot dashed (dashed) curve: result with the spin-flip occurring only for the interacting $u(d)$ quark (as in Ref. [33]). Right panel: the same for the MIT Bag model

Fig. 5: The same of Fig. 4, but for the Boer-Mulders function. 


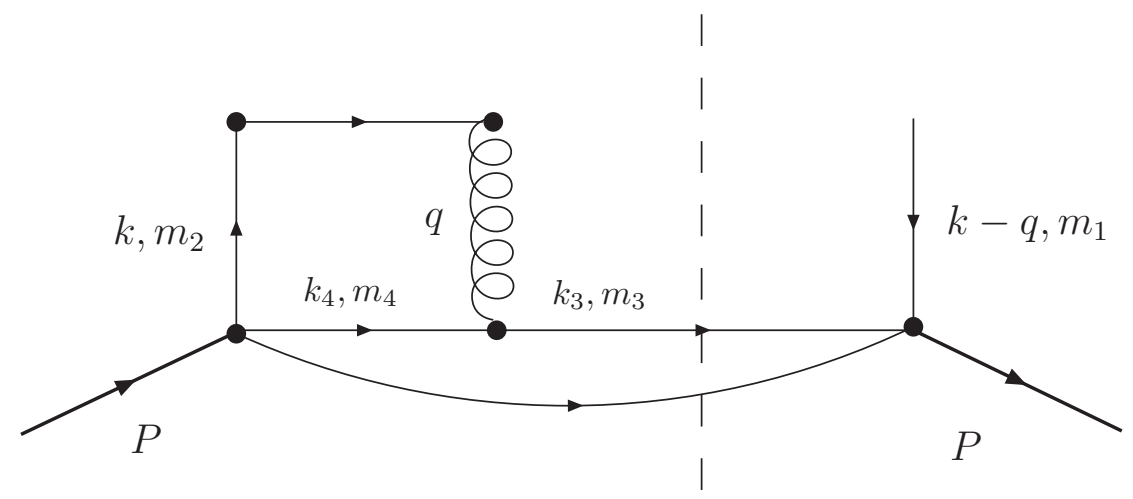

FIG. 1: 

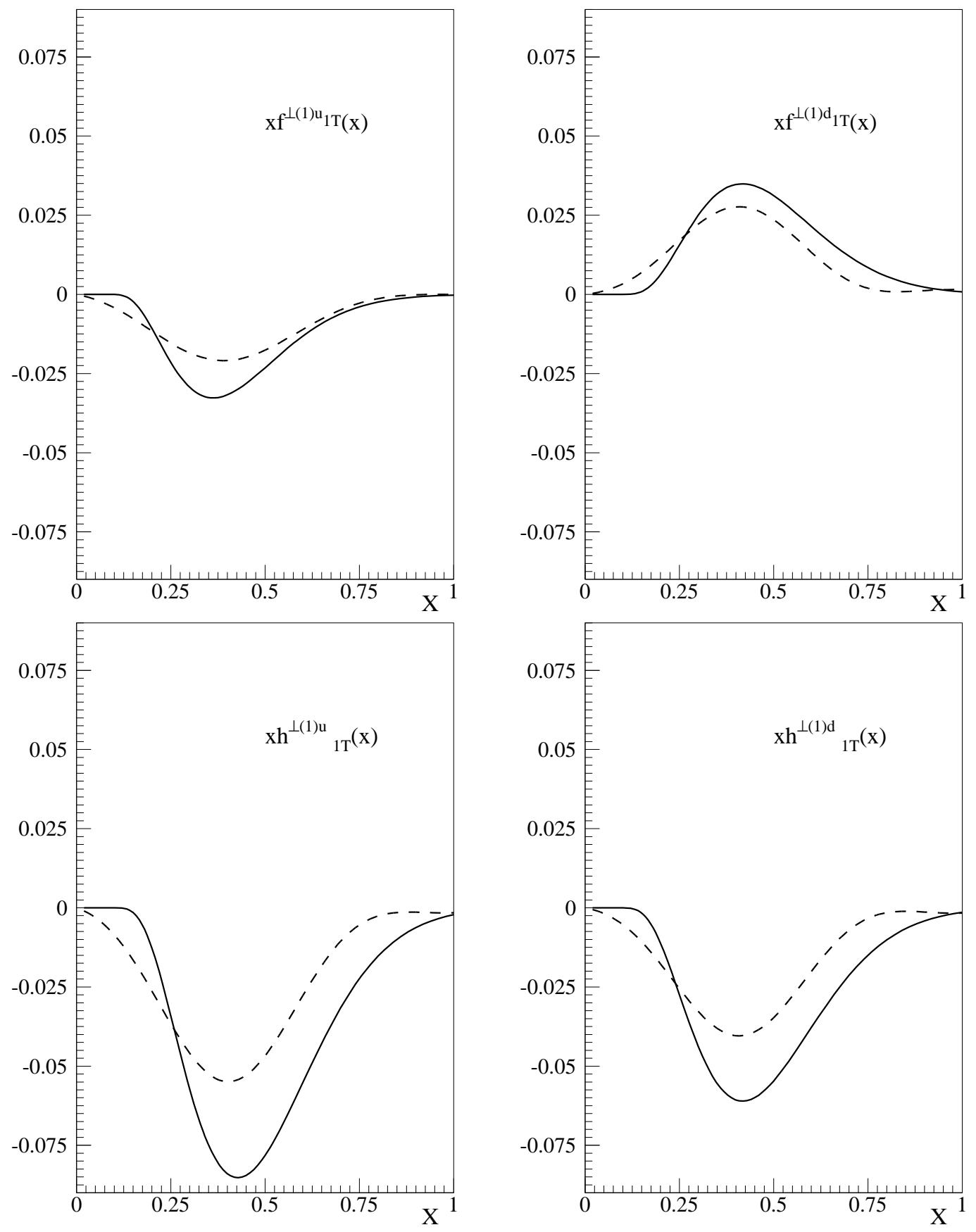

FIG. 2: 

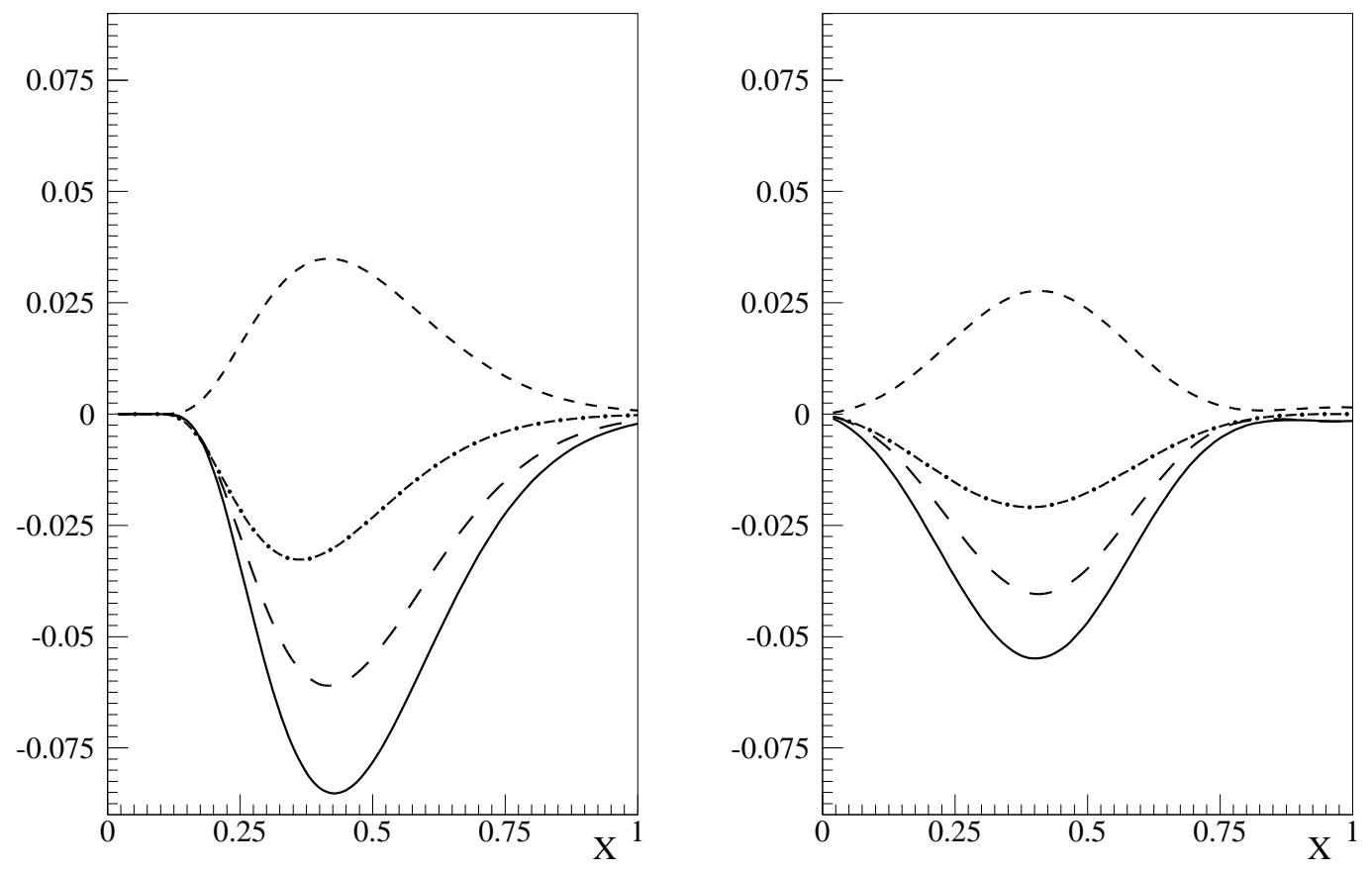

FIG. 3: 

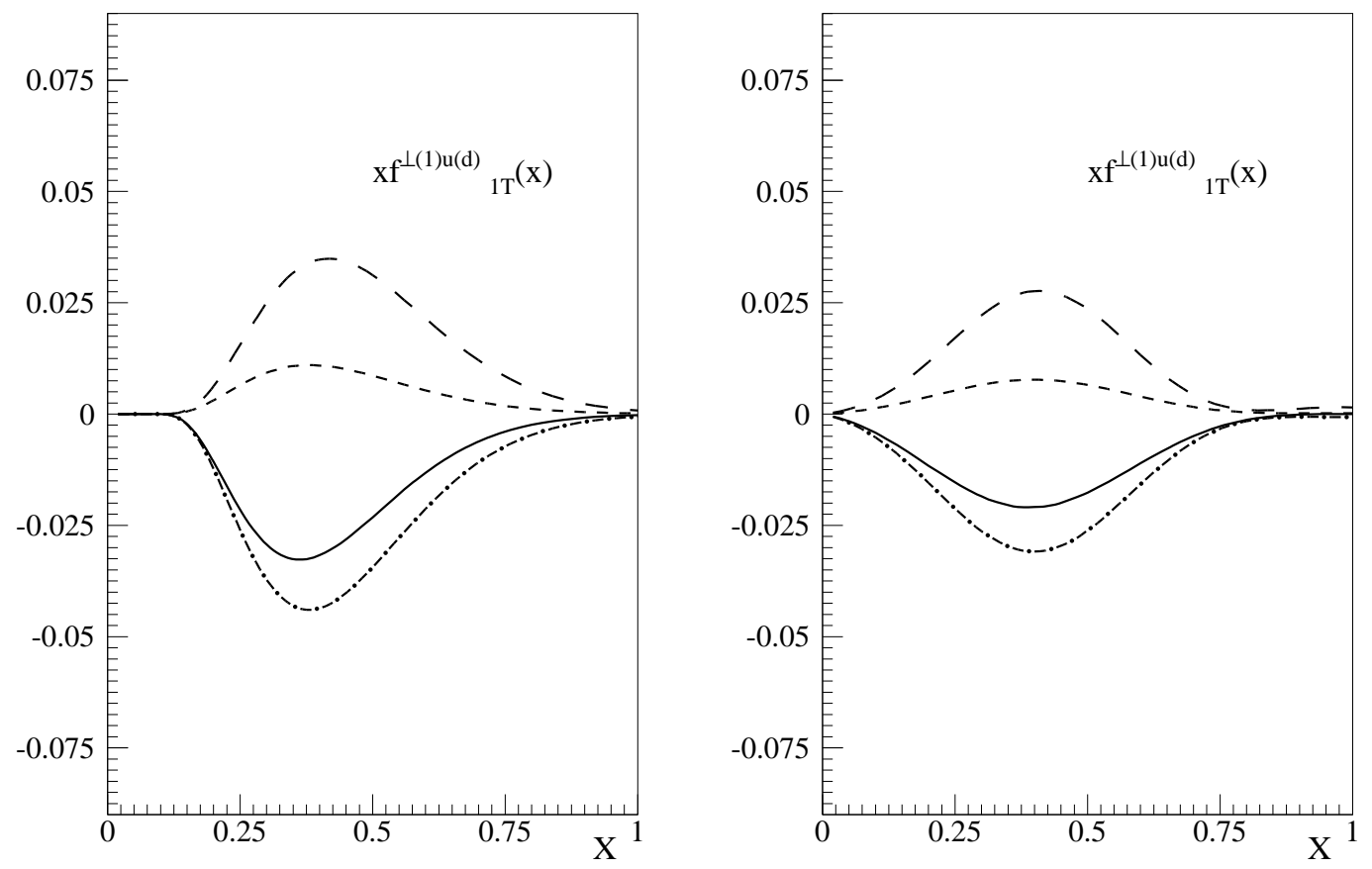

FIG. 4: 

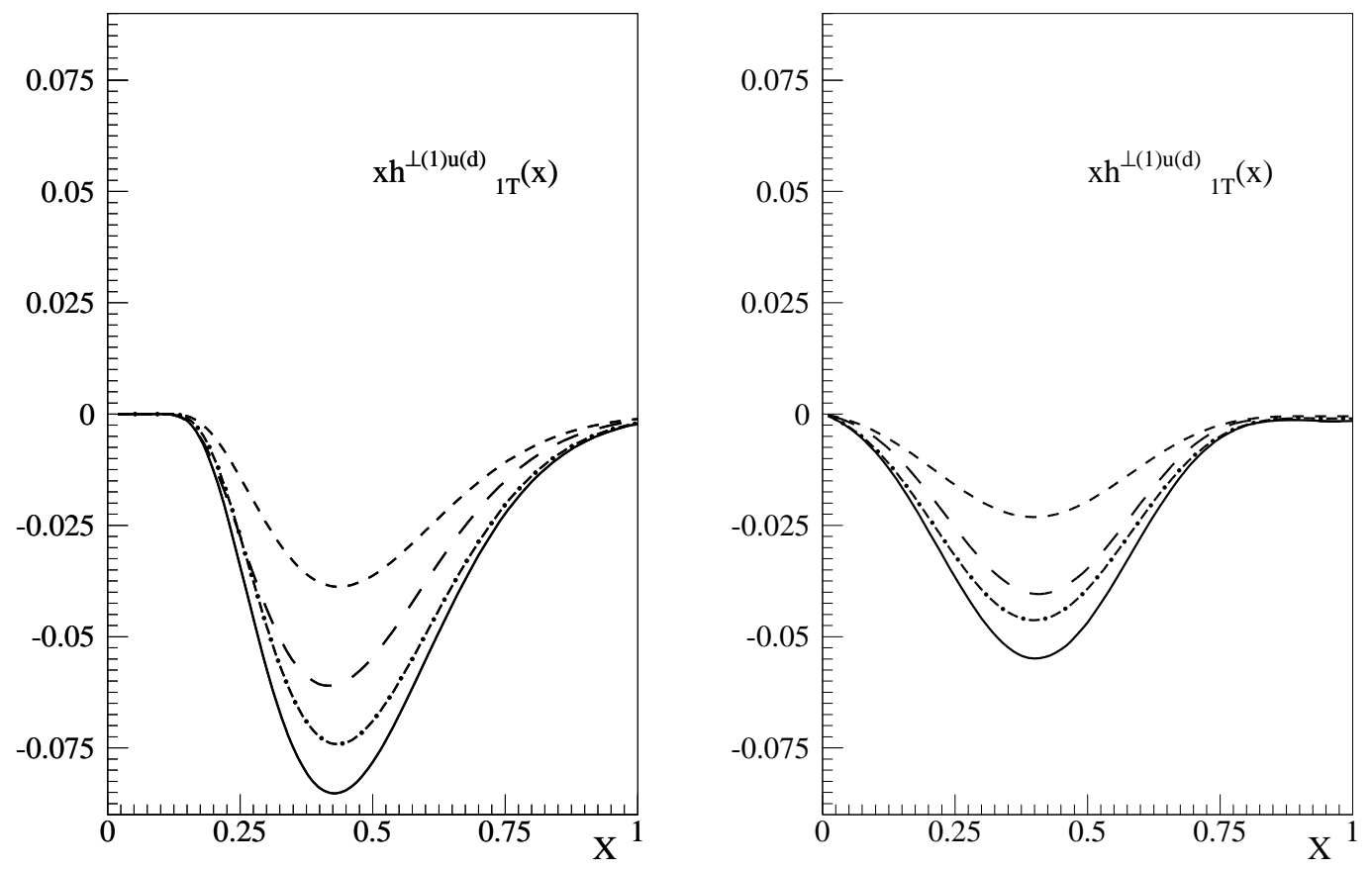

FIG. 5: 